\title{
Combined shear/tension testing of fibre composites at high strain rates using an image-based inertial impact test
}

\author{
Lloyd Fletcher $^{1, *}$, Jared Van-Blitterswyk ${ }^{1}$, and Fabrice Pierron ${ }^{1}$ \\ ${ }^{1}$ Mechanical Engineering, Faculty of Engineering and Physical Sciences, University of Southampton
}

\begin{abstract}
Testing fibre composites off-axis has been used extensively to explore shear/tension coupling effects. However, off-axis testing at strain rates above $500 \mathrm{~s}^{-1}$ is challenging with a split Hopkinson bar apparatus. This is primarily due to the effects of inertia, which violate the assumption of stress equilibrium necessary to infer stress and strain from point measurements taken on the bars. Therefore, there is a need to develop new high strain rate test methods that do not rely on the assumptions of split Hopkinson bar analysis. Recently, a new image-based inertial impact test has been used to successfully identify the transverse modulus and tensile strength of a unidirectional composite at strain rates on the order of $2000 s^{-1}$. The image-based inertial impact test method uses a reflected compressive stress wave to generate tensile stress and failure in an impacted specimen. Thus, the purpose of this study is to modify the image-based inertial impact test method to investigate the high strain rate properties of fibre composites using an off-axis configuration. For an off-axis specimen, a combined shear/tension or shear/compression stress state will be obtained. Throughout the propagation of the stress wave, full-field displacement measurements are taken. Strain and acceleration fields are then derived from the displacement fields. The kinematic fields are then processed with the virtual fields method (VFM) to reconstruct stress averages and identify the in-plane stiffness components $G_{12}$ and $E_{22}$.
\end{abstract}

\section{Introduction}

Composite materials are increasingly used in applications that subject them to high strain rate dynamic loads. This has lead to an increased research effort to understand the strain rate dependence of composites materials. It is generally accepted that for carbon fibre composites the matrix dominated properties (transverse and shear) are strain rate dependent, whereas the fibre dominant properties show little strain rate dependence [1-3].

Current high strain rate data for the matrix dependent properties of composites has been obtained using the splitHopkinson pressure bar (SHPB) [1-6] . The SHPB technique requires that several assumptions are satisfied in order to infer the material properties from the strain gauge measurements on the bars [7]. A key assumption of the SHPB analysis is that the force on the input and output bar is equal so that that inertial effects can be neglected (i.e the specimen is in a state of quasi-static equilibrium). In order to reasonably satisfy this assumption there are strict constraints on specimen size and geometry [7]. It can be difficult to satisfy this assumption for the transverse tensile properties of a unidirectional laminate due to its brittle failure mode. As the strain at failure is small, the specimen can fail before the inertial effects have damped out. Additionally, in [5] photo-elasticity was used to show that the SHPB analysis does not allow accurate calculation of the strain for the transverse tensile properties. Consequently the strain rates in [5] were limited to below $500 s^{-1}$.
Off-axis specimens have been used in the SHPB apparatus to study the strain rate dependence of the shear properties of composites [2-4, 8]. These studies generally focus on combined compression/shear testing as this removes experimental difficulties associated with gripping the specimen. For the case of combined tension/shear loading the problems associated with inertial effects remain. This makes it difficult to populate the full compression/shear, tension/shear failure envelope with current experimental methods. Furthermore, issues associated with inertial effects limit the effective strain rates that are obtainable with current experimental techniques. Therefore, there is a need for new experimental methods that can accurately measure the matrix dominant properties of composites at high strain rates.

Recently, the Image-Based Inertial Impact (IBII) test method has emerged as a viable alternative to the SHPB technique $[9,10]$. In [10] the IBII test was shown to successfully identify the transverse modulus and strength of unidirectional carbon fibre at strain rates on the order of $2000 s^{-1}$. Therefore, the aim of this study is to extend the IBII test method to identifying the transverse and shear properties of a unidirectional composite using an off-axis configuration. This paper will focus on the identification of shear and transverse moduli at high strain rates. Future studies will address the identification of the failure envelope under combined shear/tension or shear/compression loading using the IBII method.

\footnotetext{
*e-mail: 1.c.fletcher@ soton.ac.uk
} 


\section{Theory}

Consider the off-axis composite specimen in figure 1 subjected to an impact load $F(t)$. The global co-ordinate system is denoted $(x, y)$ and the material co-ordinate system is given by $(1,2)$ with the 1 direction corresponding to the fibre direction in the composite.



Fig. 1. Schematic of an impacted off-axis composite specimen.

Here, a number of simplifying a assumptions are made: the kinematic fields are uniform through-thickness, the specimen is in a state of plane stress and that the density does not vary in space. With these assumptions and neglecting body forces, the principal of virtual work is given by:

$$
-\int_{S} \boldsymbol{\sigma}: \boldsymbol{\epsilon}^{*} d S+\int_{L} \boldsymbol{T} \cdot \boldsymbol{u}^{*} d L=\rho \int_{S} \boldsymbol{a} \cdot \boldsymbol{u}^{*} d S
$$

where $S$ is the area of the specimen and $L$ is the boundary of the area. $\boldsymbol{T}$ is the Cauchy stress vector acting on the boundary, and $\sigma$ is the Cauchy stress tensor. The virtual displacement field is given by $\boldsymbol{u}^{*}$ and the virtual strain tensor (derived from $\boldsymbol{u}^{*}$ ) is given by $\boldsymbol{\epsilon}^{*}$. The acceleration field is denoted as $\boldsymbol{a}$ and $\rho$ is the material density. The matrix and vector dot products are denoted as : and - respectively. It should be noted that all kinematic fields in this equation are functions of time and space. In order to simplify the equations here the time and space variables as function notation have been omitted.

Now, consider the following rigid body virtual field in the material co-ordinate system:

$$
\left\{\begin{array} { l } 
{ u _ { 1 } ^ { * } = 1 } \\
{ u _ { 2 } ^ { * } = 0 }
\end{array} \quad \left\{\begin{array}{l}
\varepsilon_{11}^{*}=0 \\
\varepsilon_{22}^{*}=0 \\
\varepsilon_{12}^{*}=0
\end{array}\right.\right.
$$

Substituting this virtual field into equation 1 gives the following:

$$
\int_{L} \sigma_{12} d L=\rho \int_{S} a_{1} d S
$$

Using full field measurements, the integrals in equation 3 can be approximated as discrete sums. In a similar manner to [9] and [10] the stress and acceleration integrals can be expressed as numerical averages as follows:

$$
\bar{\sigma}_{12} l=\frac{\rho S \overline{a_{1}} S}{l}
$$

where $\bar{\sigma}_{12}^{l}$ is the average shear stress over the angled section at $x_{0}$ (dashed line in figure 1 ), $S$ is the surface area of the trapezoidal shape up to the angled section (shaded area in figure 1), $l$ is the length of the angled section and $\bar{a}_{1} S$ is the average acceleration over the trapezoidal area. Equation 4 can be evaluated at any angled section $x_{0}$ provided that the section does not intersect the impacted edge as this would introduce the contribution of the impact force $F(t)$. The area $S$ can be calculated for any valid angled section $x_{0}$, using: $S=w\left[x_{0}+0.5 l \cos (\theta)\right]$. Similarly, considering a rigid body virtual field with $u_{1}^{*}=0$ and $u_{2}^{*}=1$ (null virtual strains) yields the following result for the average transverse stress:

$$
\bar{\sigma}_{22} l=\frac{\rho S{\overline{a_{2}}}^{S}}{l}
$$

where the overline notation indicates averages as defined in equation 4. Equations 4 and 5 can be used to plot stress-strain curves for stiffness identification. For equation 4 a shear stress-strain curve can be plotted using the stress average $\overline{\sigma_{12}} l$ and strain average $\overline{\varepsilon_{12}} l$. Linearly fitting this curve will yield the shear modulus $G_{12}$. A stressstrain curve for the transverse modulus can be obtained by neglecting the contribution of the fibre direction strains using the assumptions outlined in [10] section 2.3. For this case $\overline{\sigma_{22}} l$ is plotted against $\overline{\varepsilon_{22}} l$ and linearly fitted to obtain $E_{22}$.

\section{Experiment methodology}

\subsection{Apparatus}

The carbon fibre material used for this study is a low temperature cure, uni-directional pre-preg tape, Gurit SE70. A unidirectional plate was made from 12 layers of pre-preg $\left([0]_{12}\right)$. The plate had a cured thickness of $3.72 \mathrm{~mm}$. A specimen of $70 \times 43 \mathrm{~mm}$ was cut from the plate using an irrigated diamond blade at $45^{\circ}$ to the fibre direction. These dimensions were selected to allow for maximum spatial resolution of the camera. These dimensions also allow for $2 \mathrm{~mm}$ of rigid body translation throughout the impact test without the specimen moving out of frame. The density of the material was measured to be $1530 \pm 25 \mathrm{~kg} \cdot \mathrm{m}^{-3}$.

The specimens were impact tested using a purpose-built gas gun. A close-up photograph of the main experimental components is shown in figure 2. The gas gun and a impact chamber are shown in figure 3 . The projectile was a $45 \mathrm{~mm}$ diameter aluminium 6061-T6 cylinder that was $25 \mathrm{~mm}$ long. The projectile was encased in a nylon sabot to reduce friction with the gas gun barrel. The impact speed was measured to be $50 \mathrm{~m} . \mathrm{s}^{-1}$ using two infra-red light gates mounted at the barrel exit. The specimen was bonded to a cylindrical waveguide made from the same material as the projectile. The purpose of the waveguide was to reduce the effects of misalignment on the stress wave input to the specimen. The waveguide was mounted 
on a wedge shaped foam stand in front of the gas gun barrel.

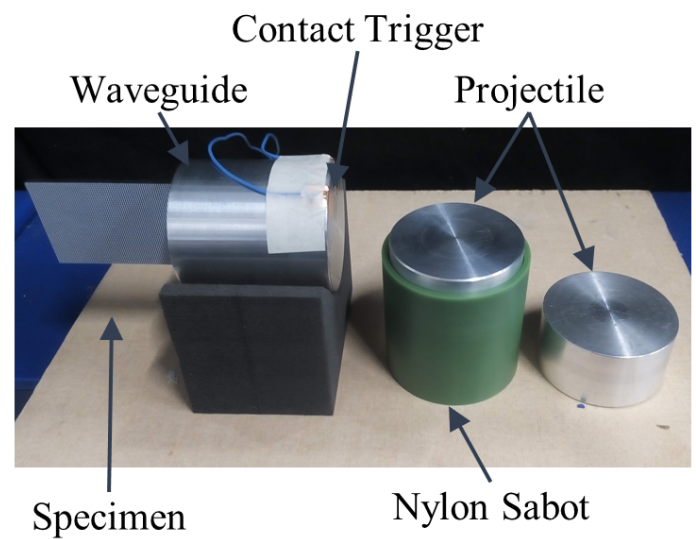

Fig. 2. Close-up view of the main experimental components: projectile, waveguide and sabot.

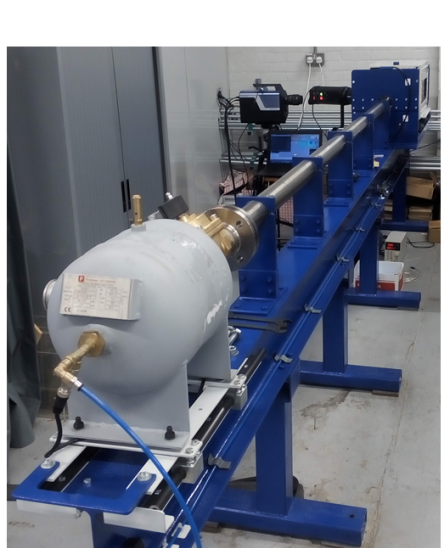

(a) Gas Gun

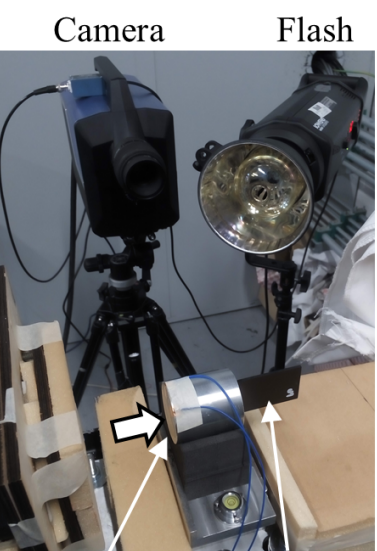

Impact Face Specimen

(b) Impact Chamber
Fig. 3. (a) Gas gun impact test rig and (b) close up view of the impact chamber showing the specimen in-situ with the camera and flash.

Throughout the impact test the specimen was imaged using a Shimadzu HPV-X camera with a frame rate of $2 \mathrm{Mfps}$ (integration time of $110 \mathrm{~ns}$ ). A total of $128 \mathrm{im}-$ ages were taken at a resolution of $400 \times 250$ pixels. A copper contact trigger on the front of the waveguide was used to trigger the camera, including a delay to account for the wave propagation time through the waveguide into the specimen.

A grid was printed directly onto the sample using a Canon Oce Arizona 1260XT flatbed UV inkjet printer. As the sample was black in colour the grids were printed as a series of white squares. The grids had a pitch of $0.9 \mathrm{~mm}$ and were sampled at 5 pixels per period. A Bowens Gemini 1000 Pro flash was used for lighting during the impact test. As the flash has a rise time of $110 \mu \mathrm{s}$ it was triggered independently from the camera using the light gates and a custom built Arduino system.

\subsection{Data processing}

The grid images were processed to extract the displacement fields using a freely available code, see [11]. The displacement fields were then post-processed using a custom Matlab code to obtain strain and accelerations fields using the centred finite difference method. As the displacement fields contain noise it was necessary to smooth the data prior to differentiation. In space, a Gaussian filter was used with a kernal length of 35 pixels. In time, a 3rd order Savitsky-Golay filter was used over 13 frames. An image deformation software pipeline is currently being developed to select optimal smoothing parameters and estimate errors in manner similar to $[12,13]$, this will be completed as future work.

All kinematic fields were initially obtained in the global $(x, y)$ co-ordinate system. These were then rotated to the material co-ordinate system using the corresponding rotation matrix for vectors or tensors respectively. The average strains along each angled section $\left(\bar{\varepsilon}_{12} l\right.$ and ${\overline{\varepsilon_{22}}}^{l})$ were obtained from the strain fields rotated to the material coordinate system. As the strain measurement points were arranged in a grid pattern in the global co-ordinate system the fields were linearly interpolated along the co-ordinates of the angled section using the the Matlab function 'scatteredinterpolant'. The acceleration fields were then processed using equations 4 and 5 to obtain the average shear and transverse stress at each angled section as a function of time. The stress-strain curves at each angled slice along the specimen length were then plotted and linearly fitted to obtain the shear and transverse modulus.

\section{Results and discussion}

The stress-strain curves for the average shear stress ${\overline{\sigma_{12}}}^{l}$ and strain $\overline{\varepsilon_{12}}$ are shown in figure 4 for several sections along the length of the specimen. The stress-strain curves show linear elastic behaviour and are consistent between sections. The shear modulus identified for every angled section is shown in figure 5. Taking the average over all sections gives an identified shear modulus of $G_{12}=3.4 \mathrm{GPa}$. The peak average shear strain rate was: $\bar{\varepsilon}_{12}^{l}$ max $=1300 s^{-1}$. The identified shear modulus seems low compared to quasi-static values given in the literature for other carbon composites, see the summary tables in [2].

Given that the strain rate here is quite high it is expected that the shear modulus will increase relative to the quasistatic value. Unfortunately, quasi-static reference data was not available for the shear modulus of this material so it is not possible to comment on strain rate effects at this stage. It should also be noted that the composite material in this study uses an a-typical low temperature cure resin so direct comparison with quasi-static data for this particular composite system is required. A more meaningful comparison can be made with the transverse modulus as this was tested at both quasi-static and high strain rates for this specific material in [10]. 

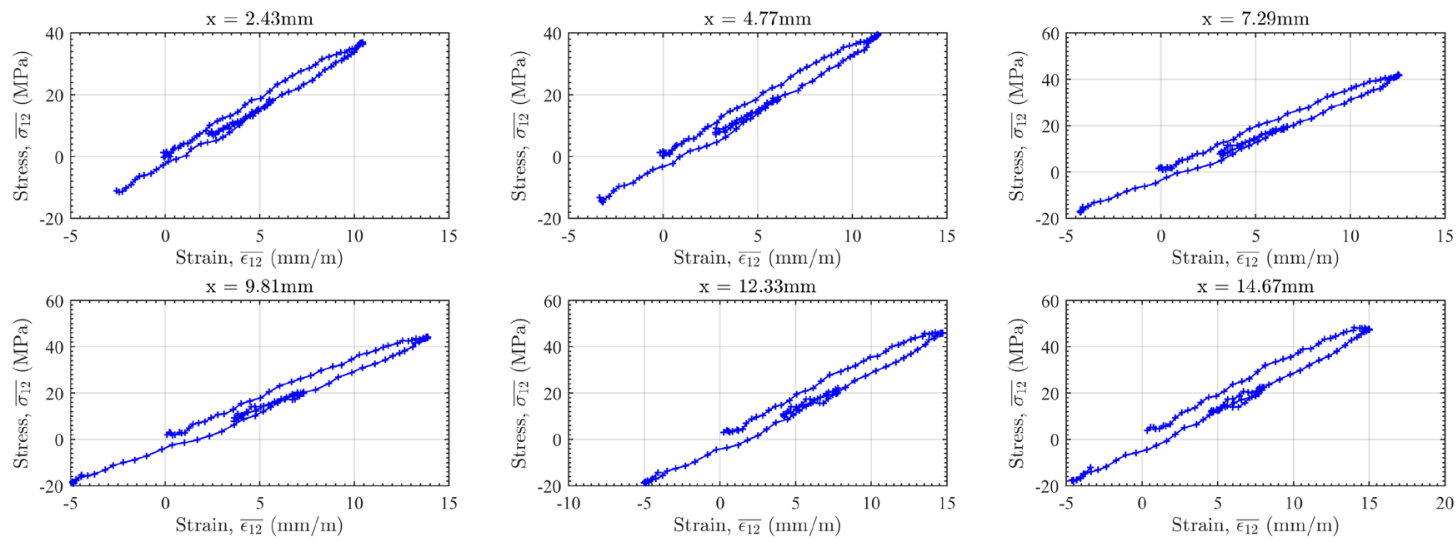

Fig. 4. Shear stress-strain curves at several angled sections along the specimen length.

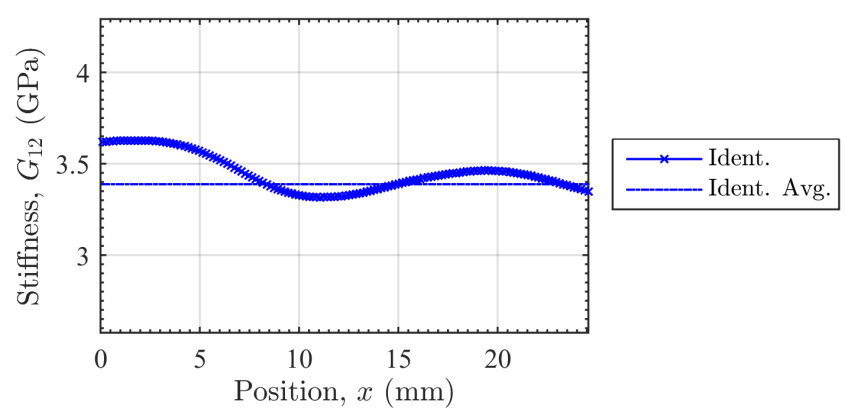

Fig. 5. Identified shear modulus over each angled section of the specimen.

The stress-strain curves for the average transverse stress and strain are shown in figure 6 for several sections along the specimen length. The transverse modulus identified at each of the angled sections is shown in figure 7. Taking the average over all angled sections gives an identified transverse modulus of $7.8 \mathrm{GPa}$. For this off-axis test specimen the peak strain rate in the transverse direction was: $\overline{\varepsilon_{22}}$ max $=820 \mathrm{~s}^{-1}$. This result compares extremely well to a previous study on the same material [10]. The quasi-static reference modulus in [10] was found to be $7.29 \pm 0.09 \mathrm{GPa}$ while the transverse modulus was found to be $7.9 \pm 0.3 \mathrm{GPa}$ at a nominal strain rate of $2000 \mathrm{~s}^{-1}$.

\section{Conclusion and future work}

The Image-Based Impact test was successfully used to identify the shear and transverse modulus of a unidirectional composite at high strain rates (on the order $\left.1000 s^{-1}\right)$. For the material tested, the identified shear modulus was $G_{12}=3.4 \mathrm{GPa}$ and the transverse modulus was $E_{22}=7.8 \mathrm{GPa}$. This demonstrates the use of the IBII test to obtain multiple orthotropic stiffness parameters in a single high strain rate test. Future work will include testing off-axis specimens at multiple angles to identify all inplane stiffness components for a uni-directional laminate using the virtual fields method. The purpose of this will be to determine the minimum number of tests required to identify all orthotropic stiffness components using the IBII test. These tests will also be used to develop a method of strength identification at high strain rates using the IBII test. This will allow for the construction of a shear/tension, shear/compression failure envelope. Finally, image deformation simulations will also be conducted to identify optimal smoothing parameters and quantify uncertainties, in a similar manner to $[12,13]$.

\section{References}

1. A. Gilat, R.K. Goldberg, G.D. Roberts, Composites Science and Technology 62, 1469 (2002)

2. H.L. Gowtham, J.R. Pothnis, G. Ravikumar, N.K. Naik, Polymer Testing 32, 1334 (2013)

3. H. Koerber, J. Xavier, P.P. Camanho, Mechanics of Materials 42, 1004 (2010)

4. H. Cui, D. Thomson, A. Pellegrino, J. Wiegand, N. Petrinic, Composites Science and Technology $\mathbf{1 3 5}$, 106 (2016)

5. L. Melin, L. Asp, Composites Part A: Applied Science and Manufacturing 30, 305 (1999)

6. H.M. Hsiao, I.M. Daniel, R.D. Cordes, Journal of Composite Materials 33, 1620 (1999)

7. B.A. Gama, S.L. Lopatnikov, J. Gillespie, John W, Applied Mechanics Reviews 57, 223 (2004)

8. J.L. Tsai, C.T. Sun, Composites Science and Technology 65, 1941 (2005)

9. F. Pierron, H. Zhu, C. Siviour, Philosophical Transactions of the Royal Society of London A: Mathematical, Physical and Engineering Sciences 372, 20130195 (2014)

10. L. Fletcher, J. Van-Blitterswyk, F. Pierron, Composites Part A: Applied Science and Manufacturing (2018), submitted

11. M. Grédiac, F. Sur, B. Blaysat, Strain 52, 205 (2016)

12. B. Lukić, D. Saletti, P. Forquin, Phil. Trans. R. Soc. A 375, 20160168 (2017)

13. M. Rossi, P. Lava, F. Pierron, D. Debruyne, M. Sasso, Strain 51, 206 (2015) 

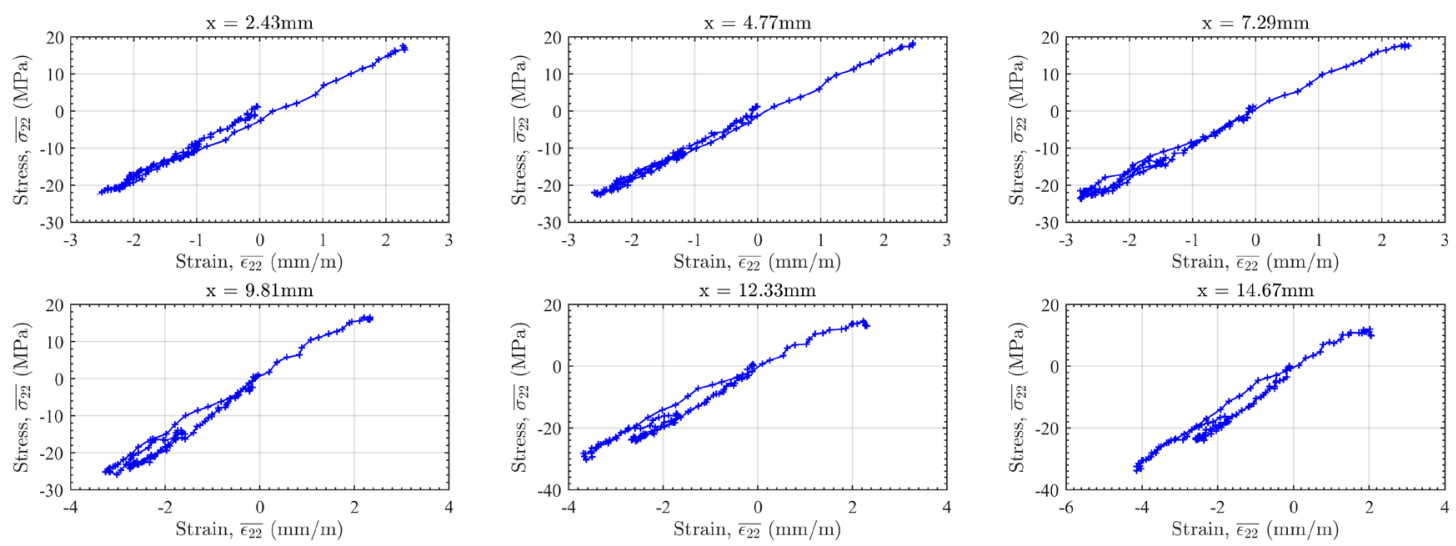

Fig. 6. Transverse stress-strain curves at several angled sections along the specimen length.

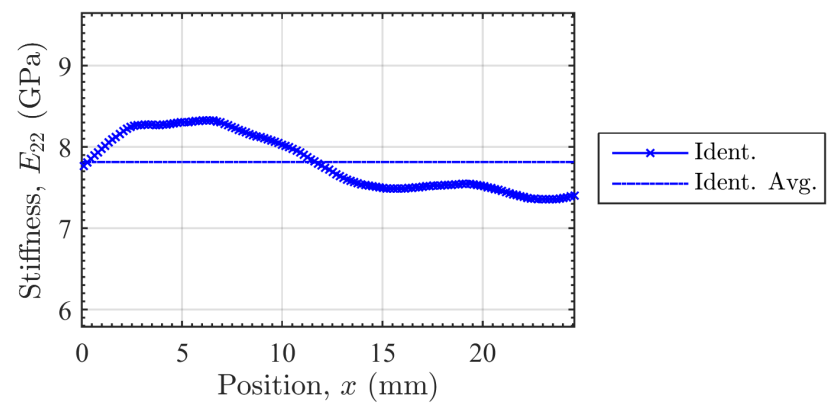

Fig. 7. Identified transverse modulus over each angled section of the specimen. 
